\title{
1 Instability of common beans during storage causes hardening: the role of glass \\ 2 transition phenomena
}

3 Claire M. Chigwedere ${ }^{a^{*}}$, Jessika N. Humerez Flores ${ }^{\mathrm{a}}$, Agnese Panozzo ${ }^{\mathrm{a}}$, Ann M. Van Loey ${ }^{\mathrm{a}}$ and

4 Marc E. Hendrickx ${ }^{a}$.

5 Corresponding author post-publication: clrchigwedere1@gmail.com

marceg.hendrickx@kuleuven.be

$7 \quad{ }^{*}$ Tel.: +3216321572

8 aKU Leuven, Laboratory of Food Technology, Leuven Food Science and Nutrition Research

9 Center (LFoRCe), Department of Microbial and Molecular Systems ( $\left.\mathrm{M}^{2} \mathrm{~S}\right)$, Kasteelpark Arenberg

1022 Box 2457, 3001, Heverlee, Belgium.

11 Chemical compounds studied in this article

12 Phosphorus pentoxide _ PubChem CID 14812

13 Potassium hydroxide_ PubChem CID 14797

14 Sodium iodide_PubChem CID 5238

15 Potassium iodide_PubChem CID 4875

16 Sodium bromide_PubChem CID 253881

17 Ammonium sulphate_PubChem CID 6097028

18 Potassium sulphate_PubChem CID 24507

19 Potassium chloride_PubChem CID 4873

20 Natamycin_PubChem CID 5284447

21 Microcrystalline cellulose_PubChem CID 62698

\section{Abstract}

Long-term storage of common beans leads to loss of cooking quality and an ill-defined solution, appropriate storage, is recommended. Therefore, the polymer science theory of glasses that hypothesizes stability of a system below its glass transition temperature $\left(T_{g}\right)$ was applied to determine bean stability during storage in relation to cooking behavior. Since composition influences $T_{g}$, powders of cotyledons and seed coats in addition to whole beans were equilibrated above different saturated salt solutions in order to generate materials with different moisture contents. A thermal mechanical compression test which measures compressibility changes in a system upon reaching its glass-rubber transition temperature region was conducted to obtain the $T_{g}$. A $T_{g}$-moisture relation was established, whose relevance was confirmed by storage and cooking experiments which showed development of hard-to-cook in

34 beans stored above $T_{g}$ but not below it. Therefore, this relation constitutes a stability map for 35 storage of common beans. 


\section{Keywords}

37 Common beans; Cotyledons; Seed coats; Stability; Glass transition temperature; Moisture 38 content

39

40

41

42

\section{Introduction}

The postharvest state of seeds that is characterized by a time-dependent deterioration process leading to loss of viability and eventual mortality is termed aging (Walters, 1998). Hydrolytic and oxidative reactions are both a cause and consequence of aging and include lipid and protein degradation, damage of membranes, loss of enzyme activity, accumulation of mutations in chromosomes and nucleic acids damage (Murthy et al., 2003; Walters, 1998). According to Walters et al. (2005), longevity of seeds is hereditary and the rate of degradation and progression towards death is exacerbated by storage conditions to which the seeds are exposed. Legume seeds are no exception to this and it has been known for long that most of them undergo physiological and/or biochemical changes during storage. In particular, common beans (Phaseolus vulgaris) may undergo color changes in colored seed coats (Edmister et al., 1990), partial or complete water impermeability of the seed coat (termed hard shell) and hardening of cotyledons (termed hard-to-cook) (El-Tabey Shehata, 1992) among others. Attempts to decipher some of these changes have yielded no more than a list of postulations put forward as mechanisms through which they occur. Factors that influence their occurrence can be grouped into agronomic conditions, such as mineral composition and content of the soil (Paredes-López et al., 1989), intrinsic characteristics of the seeds, such as variety differences, environmental conditions (temperature and relative humidity) under which the seeds are stored, and storage time (Njoroge et al., 2015).

Regardless of composition, food systems can be stored under conditions which greatly delay degradation phenomena thereby prolonging their shelf life. In this context, the glass transition theory can provide a very useful approach (Roos, 2010). When food matrices undergo a fast decrease in temperature, the aqueous phase becomes very viscous, eventually assuming a metastable glassy state wherein mobility is infeasible except for molecular rotations and vibrations. The temperature at which the system reaches this state is the glass transition temperature $\left(T_{g}\right)$. Therefore, at temperatures below $T_{g}$, diffusion-limited reactions which are mainly responsible for degradation in foods are blocked thereby ensuring physicochemical stability of the system. 
Temperature and moisture content relations that ensure stability of food systems can be established by determination of $T_{g}$ values of a food system containing different quantities of water at a constant temperature. To the best of our knowledge, the concept of $T_{g}$ has been studied in the context of loss of viability after storage (Sun and Leopold, 1994; Murthy et al., 2003) but so far not in the context of aging and loss of cooking quality hence it was addressed in this study. In the aforementioned studies germination tests, as well as products of lipid peroxidation, Maillard reactions and Amadori reactions were used to assess loss of viability and monitor deterioration reactions, respectively. In the current study the development of hard-tocook (HTC) defect was selected as an indicator of the loss of cooking quality of beans. Because composition differences might influence $T_{g}$, it was imperative to analyze also bean cotyledons and seed coats separately, besides the whole bean. Whole bean, cotyledon and seed coat powders were equilibrated above different saturated salt solutions in order to generate bean materials having different moisture content. The $T_{g}$ values of these materials were determined by a thermal mechanical compression test (TMCT), based on the change in compressibility of a material in the $T_{g}$ region resulting from the transition between the glassy to the rubbery state upon heating. This approach has been used successfully before in the context of mechanical properties and processing (Boonyai et al., 2007; Pelgrom et al., 2013). The current study aims to provide well-defined appropriate conditions (temperature and moisture content) allowing for stability of common beans during storage, specifically in relation to cooking behavior, in order to prevent the development of defects and their associated consequences.

\section{Materials and methods}

\subsection{Raw Materials}

Common beans obtained from Thika Station (Kenya Agricultural and Livestock Research Organization) were sorted and cleaned to eliminate defective seeds and dirt, respectively. Since in this study, it was intended to test the hypothesis that composition differences influence $T_{g}$, the beans were peeled manually using a knife to yield cotyledons and seed coats. Cotyledons, seed coats and whole beans were milled using an IKA A10Basic mill (Germany) with incorporation of 5 ppm of an anti-mold powder, natamycin (E235), to inhibit mold growth during subsequent storage steps. Subsequently, the powders were sieved utilizing a $500 \mu \mathrm{m}$ sieve. Milling was done in duplicate hence all subsequent treatments and analyses were conducted following this duplication. The experimental approach that was utilized for this study is illustrated in Fig. 1. In 
conditioned to generate samples differing in moisture content as described in the following section.

\subsection{Generation of materials of different moisture content}

Since adsorption and desorption behavior is dissimilar, a phenomenon termed hysteresis, it was critical to ensure that only moisture adsorption occurred in the samples. To that end, the powdered whole beans, cotyledons and seed coats were first incubated above phosphorus pentoxide $\left(\mathrm{P}_{2} \mathrm{O}_{5}\right)$ for 10 days in order to condition them to a similar, very low moisture content as a starting point. Thereafter, each material was incubated above each saturated salt solution (Table 1 ) in hermetically sealed containers. Selected targeted $a_{w}$ values were in the range 0.1 to 0.99. The relative humidities of the enclosed environments corresponded to the $a_{w}$ values of the different saturated salt solutions thereby equilibrating the samples to different moisture contents. During equilibration, the materials were incubated at $4{ }^{\circ} \mathrm{C}$ as required for stability of natamycin, until attainment of equilibrium as determined gravimetrically. Incubation times depended on the $a_{w}$ of the saturated salt solution, being longer for higher $a_{w}$ values. In general, it took 3 weeks to attain equilibrium but the materials were stored for a period of at least 4 weeks to a maximum of 6 weeks.

\subsection{Characterization of bean materials}

\subsubsection{Particle size distribution}

Particle size distribution (PSD) was determined on bean materials incubated above $\mathrm{P}_{2} \mathrm{O}_{5}$ only (starting material) using an LS 13320 laser diffraction analyzer equipped with a Tornado dry powder system module (Beckman Coulter Inc., Miami, Florida, USA). Approximately $2.8 \mathrm{~g}$ of material was weighed into the measurement cell. The principle of measurement involved illumination of the sample with $750 \mathrm{~nm}$ laser light from a halogen lamp with concomitant scattering of the light by the sample particles. From the scattering patterns and based on the Fraunhofer theory, the LS 13320 software determined the PSD of the analyzed sample.

\subsubsection{Determination of moisture content and adsorption isotherms}

Moisture content of materials incubated above both $\mathrm{P}_{2} \mathrm{O}_{5}$ and the different saturated salt solutions was determined gravimetrically using the oven-drying method following a procedure described by Chigwedere et al. (2018). Duran borosilicate bottles that were preheated in a Jouan EB 115 (Thermo Electron Corporation, USA) oven at $103^{\circ} \mathrm{C}$ for $1 \mathrm{~h}$ were cooled in a desiccator before approximately $2 \mathrm{~g}$ of bean materials was weighed into them. The materials were dried at $103^{\circ} \mathrm{C}$ for $20 \mathrm{~h}$ and moisture content was calculated as weight loss from the 
samples. Moisture content was expressed on dry basis and thereafter, adsorption isotherms, which describe the water uptake behavior of materials, were constructed (moisture content vs $\left.a_{w}\right)$.

\subsubsection{Glass transition temperature measurement}

The glass transition temperature was measured using the thermal mechanical compression test (TMCT) developed by Boonyai et al. (2007). This method involves thermal scanning of a compressed material in its glassy state with change in compressibility upon reaching the $\mathrm{T}_{\mathrm{g}}$ range. Implementation and optimization of the TMCT were done following the work of Boonyai et al. (2007) with modifications in utilized equipment and measurement specifications. A modular compact rheometer (MCR302, Anton Paar, Austria) equipped with a stainless steel sample holder (cup, $\Phi=27 \mathrm{~mm}$ ) and a cylindrical custom-made flat head aluminum probe ( $\Phi=25$ $\mathrm{mm}$ ) with which a normal compression force on the sample was exerted. The dimensions of the cylinder and cup created an annulus space of $1 \mathrm{~mm}$ in the geometry, allowing for movement of the probe, and minimizing moisture exchange between the sample and its environment during measurement. Since the principle of measurement involved thermal scanning of a sample under compression, it was imperative to optimize for the thermal scanning rate. To that end, $1 \mathrm{~g}$ of skimmed milk powder was equilibrated at $10^{\circ} \mathrm{C}$ for 5 min under a $10 \mathrm{~N}$ constant normal compression force before it was increased to $30 \mathrm{~N}$ followed by thermal scanning up to $100{ }^{\circ} \mathrm{C}$ at either 1 or $2^{\circ} \mathrm{C} / \mathrm{min}$. The displacement of the probe at constant normal force $(30 \mathrm{~N})$ as a function of the temperature was detected and recorded. A Rheo Compass software (Rheo Compass 1.17, Anton Paar, Austria) was used for data collection.

Changes in compressibility of the sample would be detected by displacement of the probe thereby necessitating elimination of any other factors that would potentially induce 'false' probe displacements. Such factors included rearrangement of particles to fill air pockets amongst them and this was prevented by the equilibration phase which facilitated for any possible rearrangements to occur before thermal scanning. Another factor would be degradation of particles due to changes in physical structure under the compression force during measurement. Peleg (1983) showed that 30 N would not lead to particle degradation. Lastly, expansion of the sample holder during thermal scanning would also induce displacement of the probe. Correction for this thermal expansion was explored by thermal scanning of $1 \mathrm{~g}$ of ovendried $\left(103^{\circ} \mathrm{C}\right.$ for $1 \mathrm{~h}$ ) microcrystalline cellulose using the selected thermal scanning rate (1 ${ }^{\circ} \mathrm{C} / \mathrm{min}$ ) from the optimization mentioned earlier. Microcrystalline cellulose was oven-dried so that it would remain incompressible in the thermal scanning range explored in this study. 
Therefore, displacement of the probe during its thermal scanning was attributed solely to expansion of the sample holder and the data generated thereof was subtracted from sample data as a way of correcting for thermal expansion of the sample holder.

After optimization of the method, $\mathrm{T}_{\mathrm{g}}$ values of bean materials of different moisture contents were analyzed. One gram of material was equilibrated under a normal force of $10 \mathrm{~N}$ at $10^{\circ} \mathrm{C}$ for $5 \mathrm{~min}$ after which it underwent thermal scanning at $1{ }^{\circ} \mathrm{C} / \mathrm{min}$ under a normal force of $30 \mathrm{~N}$.

Nonetheless, the thermal scanning range depended on the expected moisture content of the sample. Samples stored at $a_{w}$ values $\leq 0.42$ were expected to have low moisture content and hence high $T_{g}$ values thus they were thermally scanned from 10 to $100{ }^{\circ} \mathrm{C}$. At higher moisture contents $\left(0.42 \leq \mathrm{a}_{\mathrm{w}} \leq 0.90\right)$, thermal scanning was from 0 to $100^{\circ} \mathrm{C}$ and at the highest moisture content explored in this study $\left(a_{w}=0.98\right)$, much lower $T_{g}$ values were expected hence thermal scanning was from -5 to $80^{\circ} \mathrm{C}$. In order to correct for thermal expansion, the dried microcrystalline cellulose was analyzed using all the different thermal scanning ranges. Analyses were done in duplicate and following data analysis (Section 2.5), plots of $T_{g}$ vs moisture content (on dry basis) were constructed for each material.

\subsection{Determination of relevance of the determined glass transition temperatures}

In order to assess the relevance of $T_{g}$ values obtained, that is, to test the hypothesis of stability of a material below its $T_{g}$, whole beans and cotyledons were stored below, around and above their $T_{g}$ values. Subsequently, through cooking experiments, their cooking behaviors were assessed for HTC development, herein considered as stability indicator. Relevance of the $\mathrm{T}_{\mathrm{g}}$ results could be proven only if materials stored below $T_{g}$ would not exhibit HTC behavior whilst those stored above it would.

\subsubsection{Storage of whole beans and cotyledons}

Certain combinations of temperatures, relative humidities and storage time have been shown to induce HTC development in common beans (Njoroge et al., 2015; Reyes-Moreno et al., 1994). In this study, whole beans and cotyledons were stored at $35^{\circ} \mathrm{C}$ for 3 months above different saturated salt solutions. The salts included sodium iodide, potassium iodide and potassium chloride, which when saturated at $35^{\circ} \mathrm{C}$ conferred relative humidities of 35,67 and $83 \%$, respectively (Greenspan, 1977). It was targeted that whole beans and cotyledons stored under these conditions would be below, around and above their $\mathrm{T}_{g}$ values, respectively. 


\subsubsection{Determination of cooking profiles}

Cooking profiles of the stored whole beans and cotyledons were determined according to a procedure described by Chigwedere et al. (2018). The materials were soaked separately in demineralized water at $25^{\circ} \mathrm{C}$ for $16 \mathrm{~h}$ in a ratio of $1: 5(\mathrm{w} / \mathrm{v})$ followed by sampling of 20 soaked, non-cooked seeds. The rest of the seeds were cooked at $95^{\circ} \mathrm{C}$, with sampling of 20 seeds every 30 min except for beans conditioned at $83 \%$ relative humidity which were sampled after $60 \mathrm{~min}$. Hardness was measured on a cotyledon from each sampled seed thereby necessitating peeling of sampled whole beans. Measurements were performed in compression mode using a TA-X2i texture analyzer (Stable Microsystems, Goldaming, England) equipped with a $25 \mathrm{~kg}$ force cell and a P25 cylindrical flat head alumina probe $(\Phi=25 \mathrm{~mm})$. The pre-test, test and post-test speeds of the probe were 2, 1 and $2 \mathrm{~mm} / \mathrm{s}$, respectively. The average of the hardness measurements per sampling time and the associated standard deviations were plotted against cooking time to generate cooking profiles. Cooking profiles of non-stored whole beans and cotyledons were generated as reference profiles for assessment of HTC development. Just after harvest, bean varieties that had $80 \%$ of bean seeds cooked within 120 min as assessed by fingerpressing tests were categorized as being easy-to-cook (Kinyanjui et al., 2015). Therefore, non-stored beans are herein considered HTC-free because their cooking satisfied the criterion stated above.

\subsection{Data modeling and analysis}

Adsorption isotherms

Adsorption isotherms were modeled by the Guggenheim, Anderson,-and de Boer (GAB) model (equation 1) using SAS version 9.3 (SAS Institute, Inc., Cary, NC, USA).

$$
M_{w}=M_{g} C K a_{w} /\left(1-K a_{w}\right)\left(1+(C-1) K a_{w}\right)
$$

The $M_{w}$ is moisture content (dry basis), $a_{w}$ is water activity and, $M_{g}, K$ and $C$ characterize sorption properties of the materials. The $\mathrm{M}_{\mathrm{g}}$ is the $\mathrm{GAB}$ moisture content corresponding to the monomolecular layer of water molecules and, $\mathrm{C}$ and $\mathrm{K}$ are energy constants related to the net heat of sorption of the monolayer and multilayers, respectively (Sablani et al., 2007).

\section{Cooking profiles}

Cooking profiles obtained from cooking experiments were modeled with modified first order kinetics (equation 2) using SAS version 9.3 (SAS Institute, Inc., Cary, NC, USA).

$$
H=H_{0} \exp (-k t)
$$


225 In equation $2, \mathrm{H}$ is the hardness of a cotyledon at any time $\mathrm{t}$ min during cooking, $\mathrm{H}_{0}$ is the initial

226 hardness, that is $\mathrm{H}$ at $\mathrm{t}=0 \mathrm{~min}$, and $\mathrm{k}$ is the softening rate constant. In order to assess the fit of

227 the model to the experimental data and the spread of residuals, the $\mathrm{R}^{2}$ adjusted and root mean

228 square error were calculated, respectively, from the obtained analysis of variance table.

229 Statistical differences among k-values were determined from 95\% confidence intervals

230 estimated by the model.

\section{Results and discussion}

\section{$232 \quad 3.1 \quad$ Particle size distribution}

233 The PSD of powdered materials equilibrated above $\mathrm{P}_{2} \mathrm{O}_{5}$ for 10 days is shown in Fig. 2. Whilst

234 whole bean and cotyledon powders showed a bimodal PSD, seed coat powder was unimodal.

235 The seed coat powder consisted largely of particles with a diameter between 100 and $1000 \mu \mathrm{m}$.

236 Although whole bean and cotyledon powders also contained similar-sized particles, their volume

237 fractions were much lower than those of seed coat particles. This means seed coat powder

238 contained very hard particles that resisted size reduction during milling unlike the other

239 materials with bimodal distributions. During maturation of legume seeds, palisade cell walls in

240 their seed coats contract leading to hardening of the seed coats (Corner, 1951). Despite whole

241 bean and cotyledon powders having bimodal PSDs, the former had a higher volume fraction of

242 larger sized particles and a lower volume fraction for the smaller-sized particles compared to

243 cotyledon powder. This difference can be attributed to presence of seed coat material in whole

244 bean powder. The seed coat constitutes $9-11 \%$ of the dry matter content of a bean seed

245 (Moraghan et al., 2006). Therefore, presence of this fraction could be the most likely reason for

246 the slight difference in PSD between whole bean powder and cotyledon powder.

\section{$247 \quad 3.2 \quad$ Moisture content and adsorption isotherms}

248 The equilibrium moisture content of bean materials incubated under different conditions of $\mathrm{a}_{w}$

249 was used to construct adsorption isotherms (Fig. 3). For all the bean materials, the equilibrium

250 moisture content values increased with increase in $\mathrm{a}_{\mathrm{w}}$. The adsorption isotherms were sigmoidal

251 and according to Vertucci \& Leopold (1987), such profiles are characteristic of legume seeds as

252 was also observed for lentil seeds by Menkov (2000). Caurie (1970) cited by Al-Muhtaseb et al.

253 (2002) proposed an explanation for the exhibition of sigmoidal sorption isotherms in foods. It

254 was suggested that above approximately $22 \%$ moisture content (dry basis), water in the food

255 system can exert and sustain vapor pressure that is close to that of pure water. Changes in $a_{w}$

256 due to decrease of moisture content below $22 \%$ moisture then give rise to the sigmoidal shape. 
257 In the current study, for each $\mathrm{a}_{\mathrm{w}}$, the equilibrium moisture content values for whole bean,

258 cotyledon and seed coat powders were similar. Therefore, they had similar adsorption isotherms

259 despite their differences in composition. At very low moisture contents, a monolayer of water

260 molecules is adsorbed onto highly hydrophilic biopolymers including proteins and

261 polysaccharides. As such, this layer is strongly bound and is characterized by an enthalpy of

262 vaporization that is higher than that of pure water (Al-Muhtaseb et al., 2002). This layer of water

263 is unavailable for physical, chemical and microbial reactions hence at such moisture content

264 values, a food system is stable. Moisture content corresponding to the monolayer water

265 molecules was estimated by fitting the GAB model to the data and all estimated parameters are

266 shown in Table 2. The moisture contents of the different bean materials corresponding to their

267 monomolecular layers of water molecules ranged from 7.3 to $8.6 \%$ and were not significantly

268 different (statistical results not shown).

269 With increase in moisture content, multilayers of water molecules bound to the matrix by 270 capillary condensation are formed above the monolayer. Unlike for strongly-bound water, these

271 layers exhibit solvent properties and can support some biochemical reactions. In this region (up

272 to $\sim 0.6 \mathrm{a}_{\mathrm{w}}$ ), moisture content is not highly dependent on $\mathrm{a}_{\mathrm{w}}$. However, further increase in

273 moisture content leads to excess water which exhibits properties of free or bulk water in the

274 system. This region of the isotherm is characterized by a high dependency of moisture content

275 on $a_{w}$ (Al-Muhtaseb et al., 2002).

276 It is surprising that in this study, at very low moisture content, the water-adsorption behavior was

277 similar for cotyledon and seed coat powders even though they have different compositions, for

278 example, protein content. Starch, which by volume and weight constitutes the majority of dry

279 matter of cotyledons did not seem to play a significant role in water adsorption probably due to

280 its non-gelatinized state.

\section{$281 \quad 3.3$ Glass transition temperature}

\section{$282 \quad 3.3 .1$ Compressibility behavior of skimmed milk powder during optimization}

283 The compressibility curves obtained during optimization of the heating rate using skimmed milk 284 powder are shown in Fig. 4. Fig. 4A shows a linear increase in displacement of the probe with 285 increased temperature up to around $35-40{ }^{\circ} \mathrm{C}$ where the slope changed followed by resumption 286 of the linear behavior. This compressibility behavior was observed at both heating rates.

287 However, $1^{\circ} \mathrm{C} / \mathrm{min}$ showed better reproducibility compared to $2^{\circ} \mathrm{C} / \mathrm{min}$, hence it was selected 288 for all subsequent analyses. Changes in compressibility are clearly demonstrated in Fig. 4B for 
the selected heating rate after correction for thermal expansion of the sample holder. This behavior can be explained in three phases; before, during and after change in slope. The first

291 phase involves simultaneous heating of the compressed powder and the sample holder which

292 undergoes thermal expansion, necessitating downward movement of the probe in order to

293 maintain the normal compression force at $30 \mathrm{~N}$. Fig. 4B shows compressibility curves being 294 superimposable with the $x$-axis during this phase meaning no net displacement. Therefore, in 295 this phase, displacement of the probe was exclusively due to thermal expansion of the sample 296 holder hence the sample was still in the glassy state. With further heating, the curves entered 297 the second phase in which they started deviating from the x-axis giving a net displacement of 298 the probe (Fig. 4B). This is because the skimmed milk powder had reached its glass-rubber 299 transition temperature range thus it became compressible, giving the compressibility curve a 300 convex shape around the transition temperature zone. In this phase, the behavior of the 301 skimmed milk powder can be described as plastic. Beyond this temperature region, the curves 302 showed linearity which is herein considered as the last phase of the compressibility behavior 303 and the powder can be described as exhibiting viscoelastic behavior. The $T_{g}$ was determined as 304 the intersection point of the tangents to the linear parts of the curve (transition region).

\section{$305 \quad$ 3.3.2 Compressibility behavior of bean materials}

306 Whole bean, cotyledon and seed coat powders that were incubated above $\mathrm{P}_{2} \mathrm{O}_{5}$ did not exhibit a glass-rubber transition within the temperature range considered for the TMCT, just like the dried microcrystalline cellulose. This is attributed to the low moisture content of the powders (2.4-

\section{$3.7 \%$, dry matter basis). Compressibility behavior of powdered bean materials of different} moisture contents exhibited similar behavior hence compressibility curves for whole bean powders only are shown in Fig. 5A. The compressibility curve for the powder with the lowest moisture content is similar to the one for skimmed milk powder (Fig. 4B) which can be considered as ideal, unlike curves for the powders with higher moisture content which deviated from the $x$-axis before the powders approached the glass-rubber transition region. This could be possibly due to changes in the ability of the particles to pack, that is, fill voids between them, as their moisture content increased. However, the change in slope of the compressibility curves (giving a convex-nature) was very evident thereby clearly showing the transition region.

318 Therefore, the $\mathrm{T}_{\mathrm{g}}$ was approximated from such compressibility curves as illustrated in Fig. 5B. At 319 higher moisture content ( $\geq 20 \%)$, the shapes of the compressibility curves deviated much further 320 from the ideal behavior by showing a concave behavior at the beginning of the analyses. These 321 distorted curves reveal that the samples were most probably above their $\mathrm{T}_{\mathrm{g}}$ already when the 
322 analyses commenced at $-5^{\circ} \mathrm{C}$. Unfortunately, this was the lowest cold condition achievable with

323 the equipment that was in use, hence all compressibility curves obtained from bean material

324 powders with moisture $\geq 20 \%$ were not considered for approximation of $T_{g}$. In addition, the

325 sample holder being exposed to ambient temperature, starting the analysis of such samples at

326 very low temperatures would pose a risk of vapor condensation on the inner walls of the cup,

327 eventually increasing the moisture content of the sample. As a result, samples with $7-16 \%$

328 moisture only were considered. Although this moisture content range is limited, it is of practical

329 relevance. In the case that common bean seeds do not dry sufficiently on the plant prior to

330 harvesting, the first postharvest unit operation is drying to around $8-10 \%$ moisture in order to

331 extend storability.

\section{$332 \quad$ 3.3.3 $\mathrm{T}_{\mathrm{g}}$-moisture relation for common beans}

333 The variation of $T_{g}$ with increase in moisture content for the different materials is shown in Fig.

334 6. For each material, $T_{g}$ analysis was conducted in duplicate on independently prepared

335 samples hence the results are shown independently for each treatment. For all materials, the

336 increase in moisture content was coupled to a decrease in $T_{g}$. This relation is attributed to the

337 mobility-enhancing effect of water, commonly referred to as plasticizing effect, in hydrophilic

338 food components. This property is facilitated by the low molecular weight of water which

339 increases the free volume of the system thus enhancing its mobility (Al-Muhtaseb et al., 2002).

340 Below $12 \%$ moisture, the $T_{g}$ values of whole bean and cotyledon powders were similar and

341 higher than those for seed coat powders. However, a tendency for similar $\mathrm{T}_{\mathrm{g}}$ values for all bean

342 materials was observed at moisture content greater than $12 \%$. These results suggest that at low

343 moisture content, dry matter composition differences may possibly significantly influence $T_{g}$

344 rather than moisture content since the compositions of whole bean and cotyledon powders are

345 comparable but very distinct from that of seed coat powders. This implies a possibility that

346 beans of a specific moisture content stored at a particular temperature can have seed coats in

347 the viscoelastic state (above $\mathrm{T}_{\mathrm{g}}$ ) whilst the cotyledons are in a glassy/elastic (below $\mathrm{T}_{\mathrm{g}}$ ) or

348 rubbery/plastic (around $\mathrm{T}_{\mathrm{g}}$ ) state. Above 12\% moisture, the $\mathrm{T}_{\mathrm{g}}$ was highly influenced by moisture

349 content and less material dependent. Since results for whole beans and cotyledons were similar

350 at all moisture contents, we can conclude that the state of cotyledons (whether below or above

$351 \mathrm{~T}_{\mathrm{g}}$ ) has a critical contribution for storage stability of whole beans. 


\subsubsection{Is the $\mathrm{T}_{\mathrm{g}}$ data obtained relevant?}

353 In order to determine whether the $T_{g}$ results obtained in this study (Section 3.3.3) are practically relevant or not, whole beans and cotyledons conditioned to different moisture contents through storage for 3 months were assessed for HTC development by performing cooking experiments (Section 2.4.). Using the moisture contents of these materials, their positions on the $\mathrm{T}_{\mathrm{g}}$-moisture graph were determined and are encircled in Fig. 6. It is clear that at the lowest moisture content $(8.1 \%)$, the whole beans and cotyledons were in a glassy state. Cotyledons with $11.7 \%$ moisture were below $T_{g}$ but whole beans with the same moisture content had seed coats around $T_{g}$ but cotyledons below $\mathrm{T}_{\mathrm{g}}$. Materials with the highest moisture content ( 15\%) were clearly above the $\mathrm{T}_{\mathrm{g}}$ of both cotyledons and seed coats and hence were in a viscoelastic state.

362 The cooking behavior (Supplementary Fig. 1) of these stored materials was assessed by 363 comparison of their softening rate constants (Table 3) in order to investigate HTC development 364 during storage. For both cotyledons and whole beans, the $\mathrm{k}$ values decreased with increase in 365 moisture content indicating a decrease in cooking quality as moisture content increased during 366 storage. Non-stored materials ( $8 \%$ moisture) were regarded as control materials. Upon cooking, cotyledons and whole beans that contained $8.1 \%$ moisture had similar softening rate constants as their corresponding non-stored materials. Therefore, their cooking behavior was similar, signifying absence of HTC in these materials. Surprisingly, the k value for cotyledons that contained $11.7 \%$ moisture was significantly lower than that of the control cotyledons. Therefore, these cotyledons developed HTC because their cooking quality deteriorated. This was an unexpected result given the position of these cotyledons in Fig. 6 which indicates that they were in a glassy state during storage. Since $T_{g}$ is a range rather than a point, it could be that these cotyledons were possibly in the vicinity of their $T_{g}$, in other words they could have been in a rubbery state.

376 Whole beans that contained the same moisture content showed different cooking behavior from 377 that of the control whole beans but their $k$ value was not significantly different from that of whole 378 beans that were stored with $8.1 \%$ moisture. This result suggests that the whole beans with $37911.7 \%$ moisture were possibly in a rubbery state during storage. Their position in Fig. 6 which 380 shows that they were above the $T_{g}$ of seed coats but below that of cotyledons could be the 381 reason for the suggested rubbery state during storage. This is an interesting result given that 382 our previous work (Chigwedere et al., 2018) showed that cotyledons significantly contribute to 383 HTC development unlike seed coats. Therefore, results from this study suggest that for storage stability of common beans in the context of their cooking quality, mobility should be infeasible in 
both cotyledons and seed coats, that is, both macrostructural components have to be in a glassy state. However, there is need to explore the mechanism by which the seed coat in a viscoelastic state would facilitate for HTC development in the bean when the cotyledons are in a glassy state. It can be hypothesised that since cotyledons consist of multiple components, some of their $T_{g}$ values might have possibly facilitated HTC development given the state of the seed coat.

Cotyledons and whole beans that had the highest moisture content during storage had the lowest $\mathrm{k}$ values which were however not significantly different from the corresponding materials containing $11.7 \%$ moisture. Their higher moisture content facilitated mobility in the matrix, leading to deteriorative reactions that could have led to the highest extent of HTC development and hence the lowest cooking quality. Biochemical reactions hypothesised as mechanistic explanations for occurrence of HTC were reviewed by El-Tabey Shehata (1992), Reyes-Moreno et al. (1993) and Liu and Bourne (1995). These results confirm the polymer science hypothesis of stability below and instability above the $T_{g}$. Therefore, the $T_{g}$ data generated in this study are relevant and the established $\mathrm{T}_{\mathrm{g}}$-moisture relation can be regarded as a stability map for postharvest storage of common beans.

\section{Conclusions}

For the first time, the present study provides, based on a material science approach, the appropriate storage conditions, that is, moisture content and temperature, that ensure storage stability of common beans in the context of aging and cooking quality. Results indicate that the hypothesis of stability below $T_{g}$ is supported as long as all the components of the beans are below their $T_{g}$ values. If this condition is not met, beans will be rendered unstable and liable to lose cooking quality. In addition, the established $\mathrm{T}_{\mathrm{g}}$-moisture relation was proven to be of practical relevance, hence it can be used as a stability map for storage of common beans. After harvest, moisture content of the beans can be determined and the temperature below which they should be stored to ensure preservation of cooking quality can be read-off from the stability map ( $T_{g}$-moisture relation). The lower the moisture content of the beans, the higher the $T_{g}$ values hence postharvest drying becomes a prerequisite for enhanced stability of beans. This is an advantage for common bean farmers in temperate regions because they can store their beans at room temperatures but not so much for those in tropical regions who may require cool conditions for bean storage during the summer period. Among the commonly consumed legumes, the susceptibility of HTC development and hence loss of cooking quality follows the 
order: common beans > Bambara groundnuts (Vigna subterranea) > cowpeas (Vigna unguiculata). Therefore, it is interesting to determine also the stability maps of Bambara groundnuts and cowpeas and other legumes in order to prevent quality deterioration during storage.

\section{Acknowledgements}

Chigwedere C. M. is a Ph.D. fellow funded by the Interfaculty Council for Development Cooperation (IRO, Grant number 000000003746). Panozzo A. is a postdoctoral researcher in the Laboratory of Food Technology who assisted with method implementation. This research did not receive any specific grant from funding agencies in the public, commercial or not-forprofit sectors.

Declarations of interest: The authors declare that they have no conflict of interest.

\section{References}

Al-Muhtaseb, A. H., McMinn, W. A. M., \& Magee, T. R. A. (2002). Moisture sorption isotherm characteristics of food products: A review. Food and Bioproducts Processing, 80, 118-128.

Boonyai, P., Howes, T., \& Bhandari, B. (2007). Instrumentation and testing of a thermal mechanical compression test for glass-rubber transition analysis of food powders. Journal of Food Engineering, 78, 1333-1342.

Chigwedere, C. M., Olaoye, T. F., Kyomugasho, C., Kermani, Z. J., Pallares, A. P., Van Loey, A. M., Grauwet, T., \& Hendrickx, M. E. (2018). Mechanistic insight into softening of Canadian wonder common beans (Phaseolus vulgaris) during cooking. Food Research International, $106,522-531$.

Corner, E. J. H. (1951). Leguminous seed. Phytomorphology, 1, 117-150.

Edmister, J. A., Breene, W. M., \& Serugendo, A. (1990). Influence of temperature, water activity and time on cookability and color of a stored Rwandan dry bean (Phaseolus vulgaris) mixture. Journal of stored products research, 26(3), 121-126.

El-Tabey Shehata, A. M. (1992). Hard-to-cook phenomenon in legumes. Food Reviews International, 8(2), 191-221.

Greenspan, L. (1977). Humidity fixed points of binary saturated aqueous solutions. Journal of Research of the National Bureau of Standards, 81(1), 89-96.

Kinyanjui, P. K., Njoroge, D. M., Makokha, A. O., Christiaens, S., Ndaka, D. S., \& Hendrickx, M. (2015). Hydration properties and texture fingerprints of easy- and hard-to-cook bean varieties. Food Science and Nutrition, 3, 39-47.

Liu, K., \& Bourne, M. C. (1995). Cellular, biological and physicochemical basis for the hard-tocook defect in legume seeds. Critical Reviews in Food Science and Nutrition, 35(4), 263298.

Menkov, N. D. (2000). Moisture sorption isotherms of lentil seeds at several temperatures. 
Journal of Food Engineering, 44, 205-211.

482

Moraghan, J. T., Etchevers, J. D., \& Padilla, J. (2006). Contrasting accumulations of calcium and magnesium in seed coats and embryos of common bean and soybean. Food Chemistry, 95, 554-561.

Murthy, U. M. N., Kumar, P. P., \& Sun, W. Q. (2003). Mechanisms of seed ageing under different storage conditions for Vigna radiata (L.) Wilczek: Lipid peroxidation, sugar hydrolysis, maillard reactions and their relationship to glass state transition. Journal of Experimental Botany, 54, 1057-1067.

Njoroge, D. M., Kinyanjui, P. K., Christiaens, S., Shpigelman, A., Makokha, A. O., Sila, D. N., \& Hendrickx, M. E. (2015). Effect of storage conditions on pectic polysaccharides in common beans (Phaseolus vulgaris) in relation to the hard-to-cook defect. Food Research International, 76, 105-113.

Paredes-López, O., Maza-Calviño, E. C., \& González-Castañeda, J. (1989). Effect of the hardening phenomenon on some physico- chemical properties of common bean. Food Chemistry, 31, 225-236.

Peleg, M. (1983). Physical characteristics of food powders. In: Peleg, M., Bagley, E.B. (Eds.), Physical properties of foods (pp. 192-323). Westport, CT: AVI Publishing Co.

Pelgrom, P. J. M., Schutyser, M. A. I., \& Boom, R. M. (2013). Thermomechanical morphology of peas and its relation to fracture behaviour. Food and Bioprocess Technology, 6, 33173325.

Reyes-Moreno, C., Paredes-Lopez, O., \& Barradas, I. (1994). A fast laboratory procedure to assess the hard-to-cook tendency of common beans varieties. Food Chemistry, 49, 187190.

Reyes-Moreno, C., Paredes-López, O., \& Gonzalez, E. (1993). Hard-to-cook phenomenon in common beans - A review. Critical Reviews in Food Science and Nutrition, 33, 227-286.

Roos, Y. H. (2010). Glass transition temperature and its relevance in food processing. Annual Review of Food Science and Technology, 1, 469-96.

Sablani, S. S., Kasapis, S., \& Rahman, M. S. (2007). Evaluating water activity and glass transition concepts for food stability. Journal of Food Engineering, 78, 266-271.

Sun, W. Q., \& Leopold, A. C. (1994). Glassy state and seed storage stability: a viability equation analysis. Annals of Botany, 74(6), 601-604.

Vertucci, C. W., \& Leopold, A. C. (1987). Oxidative processes in soybean and pea seeds: effect of light, temperature, and water content. Plant Physiology, 84, 1038-1043.

Walters, C. (1998). Understanding the mechanisms and kinetics of seed aging. Seed Science Research, 8, 223-244.

Walters, C., Hill, L. M., \& Wheeler, L. J. (2005). Dying while dry: Kinetics and mechanisms of deterioration in desiccated organisms. Integrative and Comparative Biology, 45, 751-758. 
493 Table 1: Selected salts to impart different water activities in the range 0.1 to 0.99 in enclosed

494 environments in order to generate bean materials of different moisture contents

\begin{tabular}{ll}
\hline Salt & Target $\mathbf{a}_{\mathbf{w}}$ at saturation at $\mathbf{4}^{\circ} \mathbf{C}$ \\
\hline Potassium hydroxide $(\mathrm{KOH})$ & $0.14 \pm 0.017$ \\
Sodium iodide $(\mathrm{Nal})$ & $0.42 \pm 0.099$ \\
Sodium bromide $(\mathrm{NaBr})$ & $0.64 \pm 0.072$ \\
Ammonium sulphate $\left(\left(\mathrm{NH}_{4}\right)_{2} \mathrm{SO}_{4}\right)$ & $0.82 \pm 0.068$ \\
Potassium sulphate $\left(\mathrm{K}_{2} \mathrm{SO}_{4}\right)$ & $0.98 \pm 0.091$
\end{tabular}

Table 2. Parameters estimated by the Guggenheim, Anderson, and de Boer (GAB) model for the adsorption isotherms of whole bean, cotyledon and seed coat powders. The $\mathrm{M}_{\mathrm{g}}$ is the GAB moisture content corresponding to the monomolecular layer of water molecules and, C and $\mathrm{K}$ are energy constants related to the net heat of sorption of the monolayer and multilayers, respectively. Analyses were conducted in duplicate and are presented as such.

502

\begin{tabular}{lrccc} 
Material & \multicolumn{3}{c}{$\mathbf{~}$} & $\mathbf{K}$ \\
\hline Whole beans 1 & 0.99 & $7.3 \pm 0.24$ & $123 \pm 100$ & $0.8 \pm 0.01$ \\
Whole beans 2 & 1.00 & $7.4 \pm 0.20$ & $74.3 \pm 30.6$ & $0.8 \pm 0.01$ \\
Cotyledons 1 & 1.00 & $8.6 \pm 0.44$ & $33.6 \pm 10.8$ & $0.7 \pm 0.03$ \\
Cotyledons 2 & 1.00 & $7.9 \pm 0.19$ & $66.2 \pm 20.9$ & $0.8 \pm 0.01$ \\
Seed coats 1 & 1.00 & $8.2 \pm 0.37$ & $42.9 \pm 14.9$ & $0.7 \pm 0.02$ \\
Seed coats 2 & 1.00 & $8.1 \pm 0.41$ & $42.8 \pm 17.2$ & $0.7 \pm 0.03$ \\
\hline
\end{tabular}

504

505 
508 Table 3. Parameter estimates ( \pm standard errors) for softening of whole beans and cotyledons

509 with different moisture content stored at $35^{\circ} \mathrm{C}$ for three months. The $\mathrm{k}$ is the estimated softening

510 rate constant and $\mathrm{H}_{0}$ is the normalized initial hardness.

\begin{tabular}{lcccc}
\hline \multicolumn{1}{c}{ Material } & $\mathrm{R}^{2}$ Adjusted & $\mathrm{k}^{1}\left(\mathrm{x} 10^{2}\right) \mathrm{min}^{-1}$ & $\mathrm{H}_{0}(\mathrm{~g})$ & $\mathrm{RMSE}$ \\
\hline$\%$ Moisture content of stored cotyledons & & & & \\
$7.90 \pm 0.0050$ (control) & 0.99 & $2.44 \pm 0.19^{\mathrm{a}}$ & $1.01 \pm 0.04$ & 0.041 \\
$8.10 \pm 0.0004$ & 0.99 & $1.95 \pm 0.07^{\mathrm{a}}$ & $1.00 \pm 0.02$ & 0.020 \\
$11.7 \pm 0.0001$ & 0.99 & $1.07 \pm 0.12^{\mathrm{b}}$ & $1.05 \pm 0.06$ & 0.069 \\
$15.1 \pm 0.0002$ & 0.99 & $0.76 \pm 0.03^{\mathrm{b}}$ & $1.00 \pm 0.02$ & 0.025 \\
$\%$ Moisture content of stored whole beans & & & & \\
$8.10 \pm 0.00700$ (control) & 0.99 & $1.48 \pm 0.12^{\mathrm{A}}$ & $0.99 \pm 0.04$ & 0.045 \\
$8.10 \pm 0.00006$ & 0.99 & $1.11 \pm 0.15^{\mathrm{AB}}$ & $1.06 \pm 0.07$ & 0.080 \\
$11.7 \pm 0.00020$ & 0.99 & $0.68 \pm 0.12^{\mathrm{BC}}$ & $0.92 \pm 0.07$ & 0.078 \\
$14.5 \pm 0.00000$ & 0.99 & $0.37 \pm 0.03^{\mathrm{C}}$ & $0.96 \pm 0.04$ & 0.044 \\
\hline
\end{tabular}

${ }^{1}$ Different small and capital superscript letters show significant differences among k-values for cotyledons and whole beans, respectively 


\section{$511 \quad$ Figures}

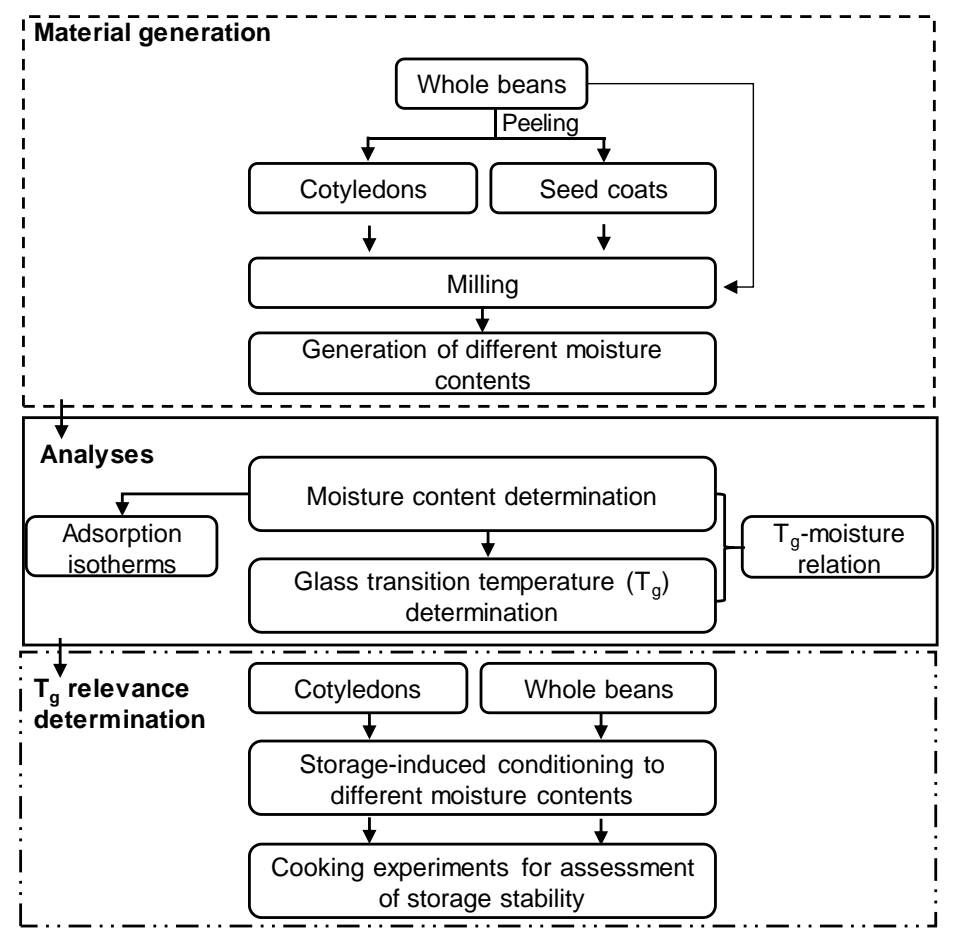

513 Fig. 1 Schematic illustration of the experimental approach of this study.

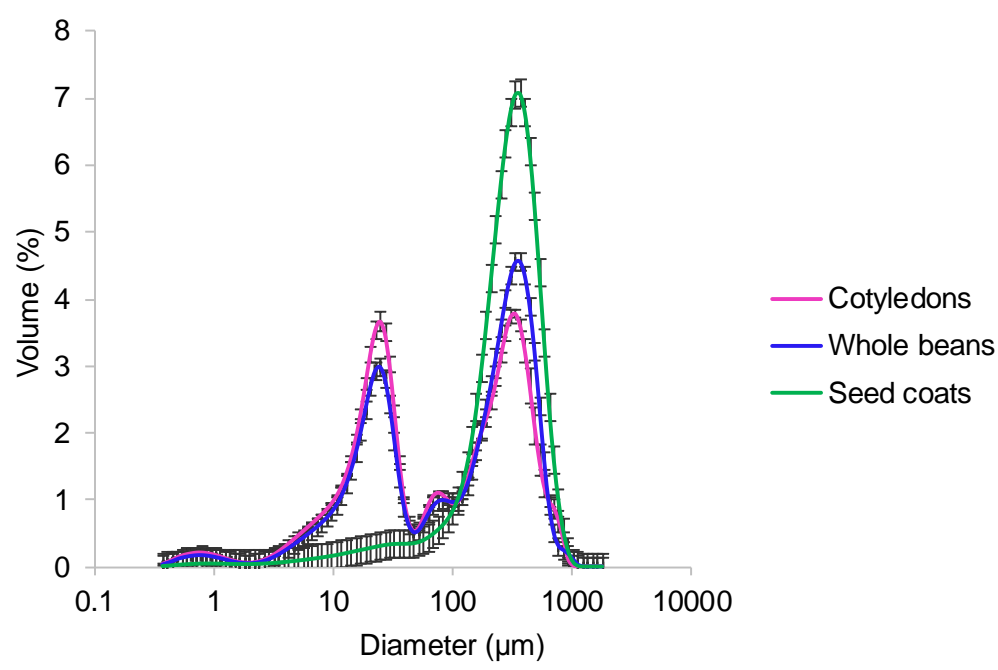

515 Fig. 2. Particle size distribution of powdered whole beans, cotyledons and seed coats after 516 equilibration above phosphorus pentoxide in order to attain the minimum possible moisture 517 content. Solid and broken lines of the same color represent duplicates of the same bean 518 material. 


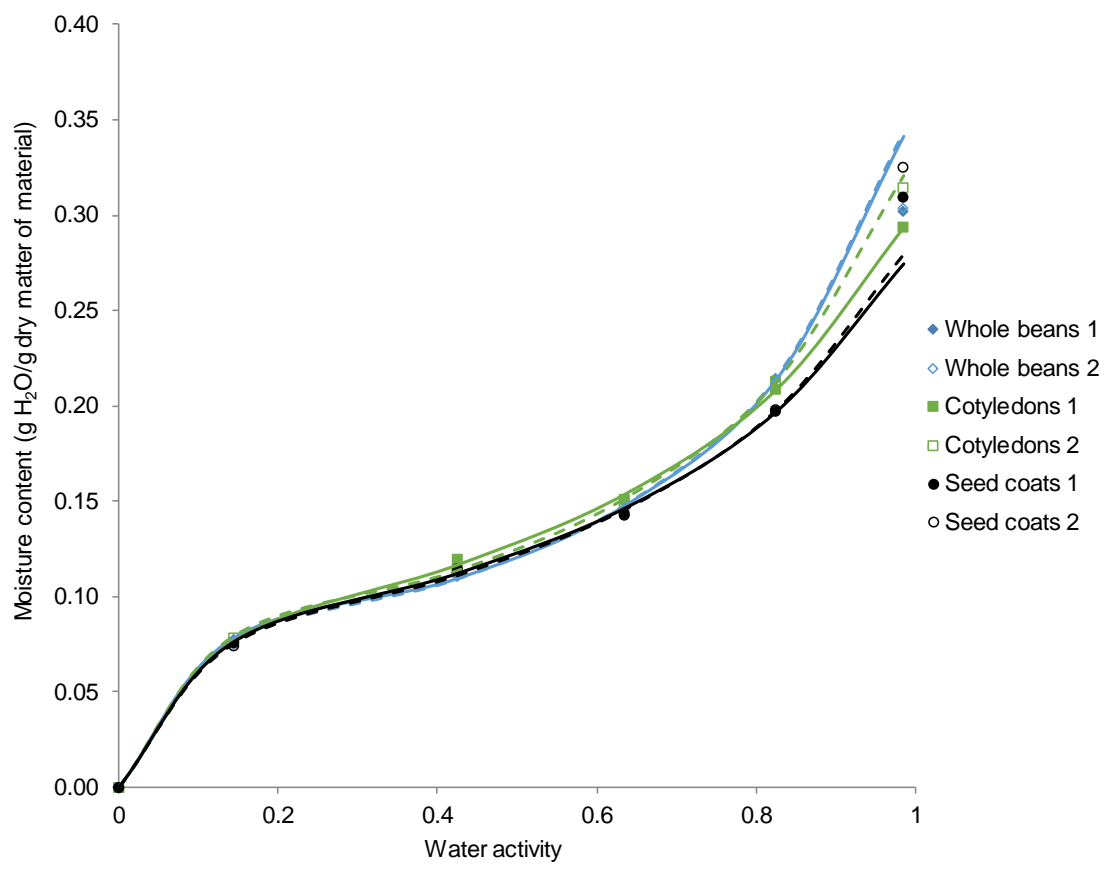

520 Fig. 3. Sorption isotherms for whole bean, cotyledon and seed coat powders at $4{ }^{\circ} \mathrm{C}$ modeled 521 with the GAB equation.
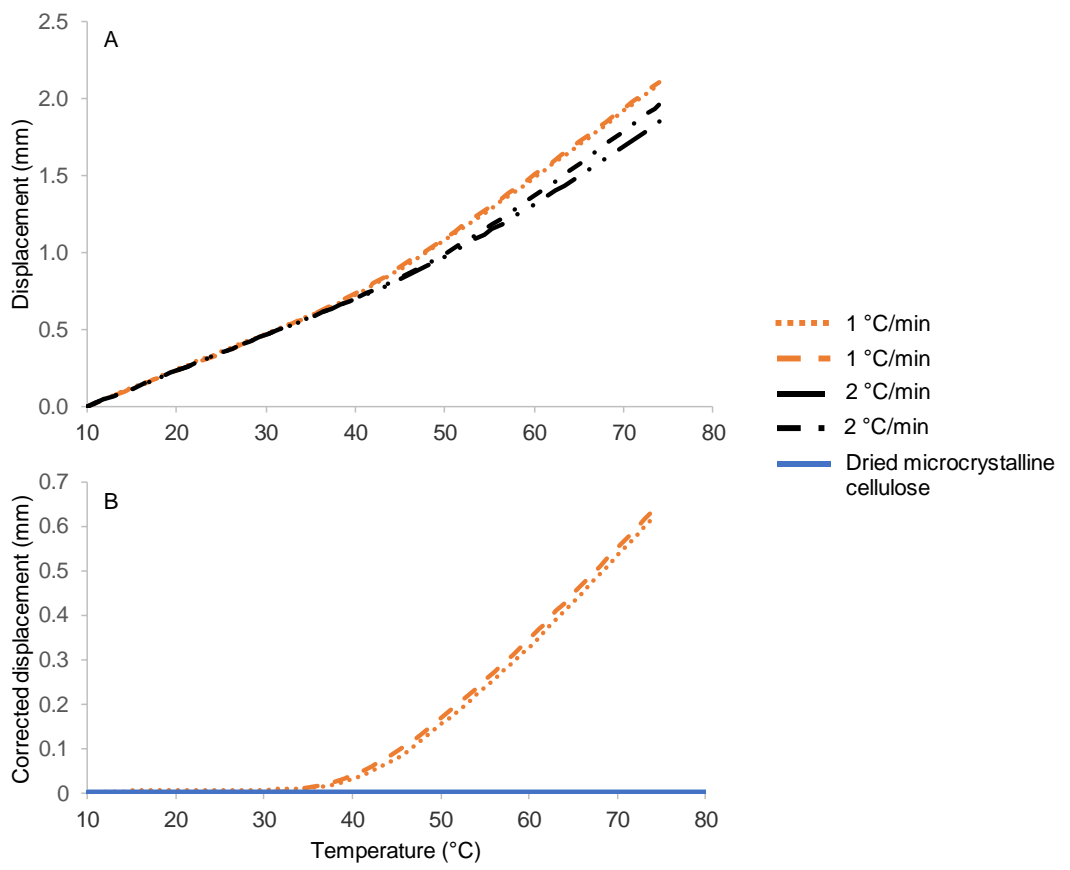

523 Fig. 4. Displacement of the probe during heating of skimmed milk powder at different heating 524 rates under a compression force of $30 \mathrm{~N}(\mathrm{~A})$ and corrected displacement for skimmed milk 525 powder at the selected heating rate $\left(1^{\circ} \mathrm{C} / \mathrm{min}\right)(\mathrm{B})$ using oven-dried microcrystalline cellulose. 

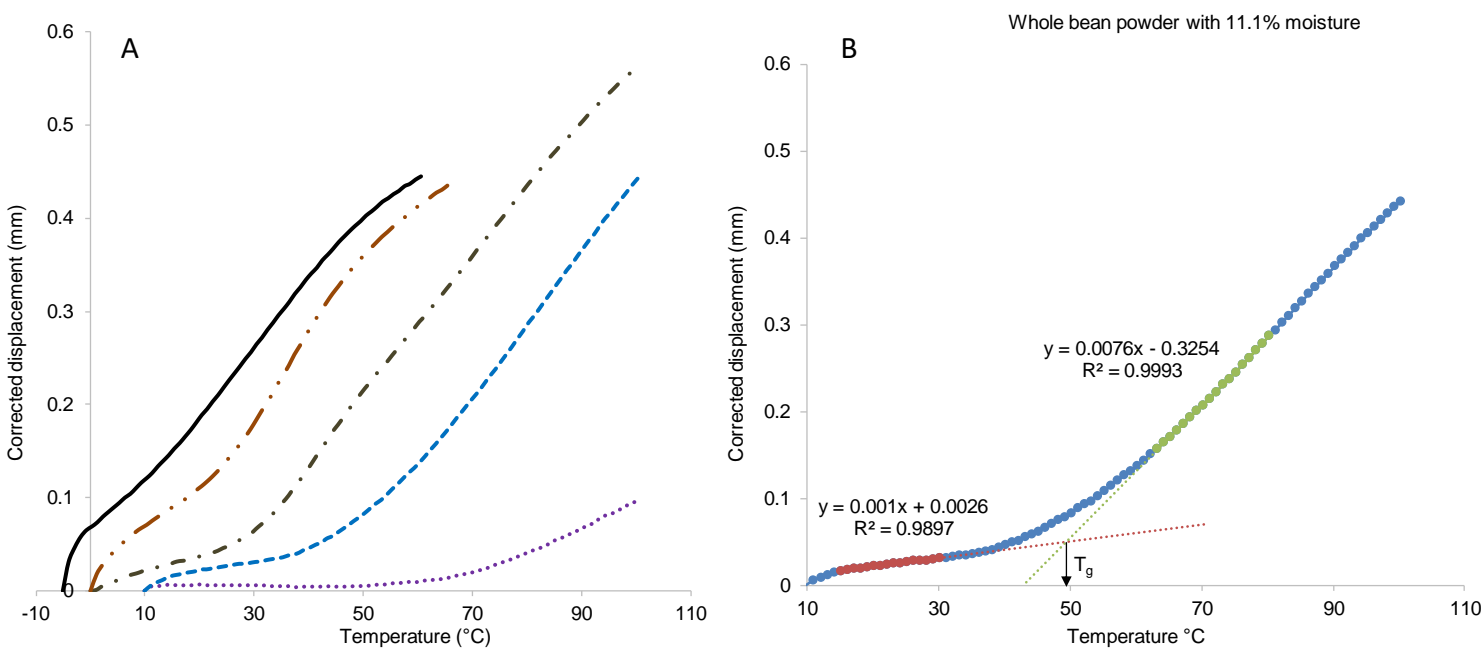

\%Moisture content

Fig. 5. Compressibility curves of whole bean powders of different moisture content $(A)$ and how

$528 \mathrm{~T}_{\mathrm{g}}$ values were obtained from them as illustrated for whole bean powder with $11.1 \%$ moisture

529 (B).

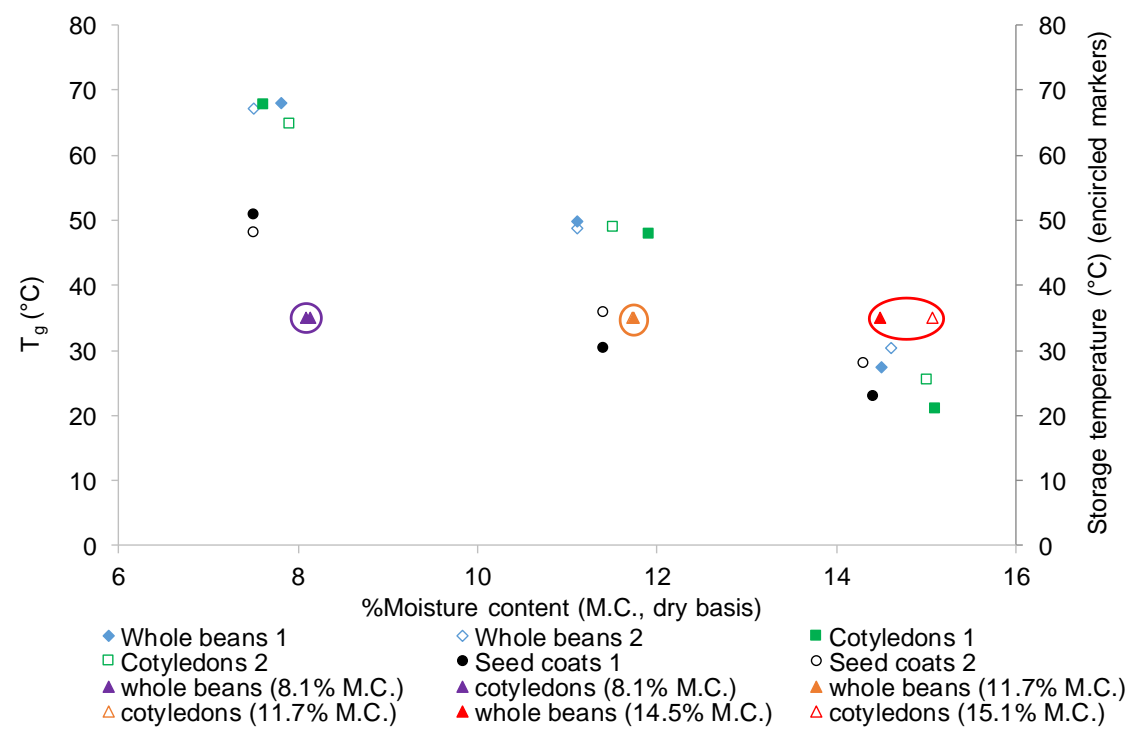

531 Fig. 6. Variation of glass transition temperature with moisture content (symbols not encircled) of

532 whole bean, cotyledon and seed coat powders. The materials were analyzed in duplicate from

533 independent treatments hence the results are presented in duplicate for each material.

534 Encircled colored triangular symbols represent the conditions of whole beans and cotyledons

535 stored at different moisture content (shown in the legend) at $35^{\circ} \mathrm{C}$ for three months in order to 536 determine relevance of $\mathrm{T}_{\mathrm{g}}$ data. 


\section{Supplementary Figure}

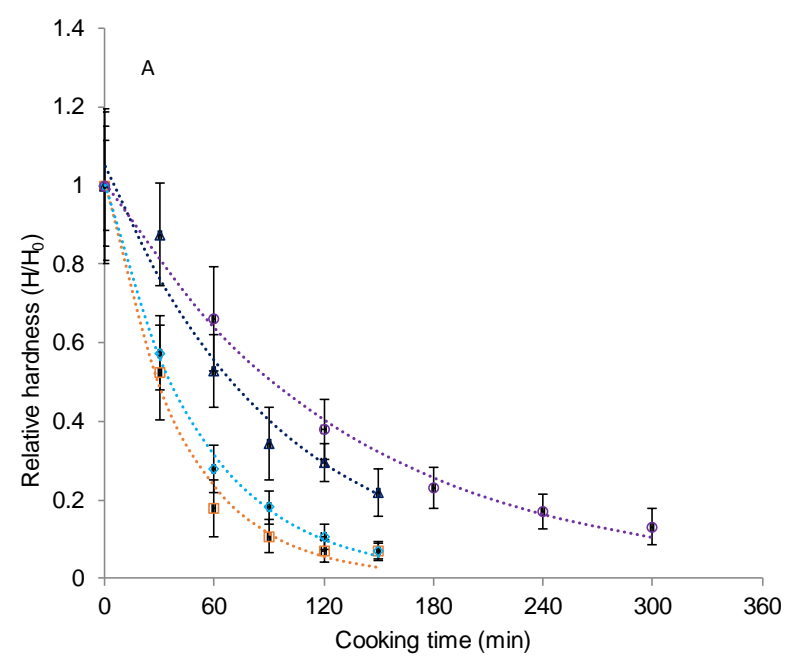

$\square$ Fresh cotyledons (control) $\triangle$ Cotyledons $(11,7 \%$ M.C)

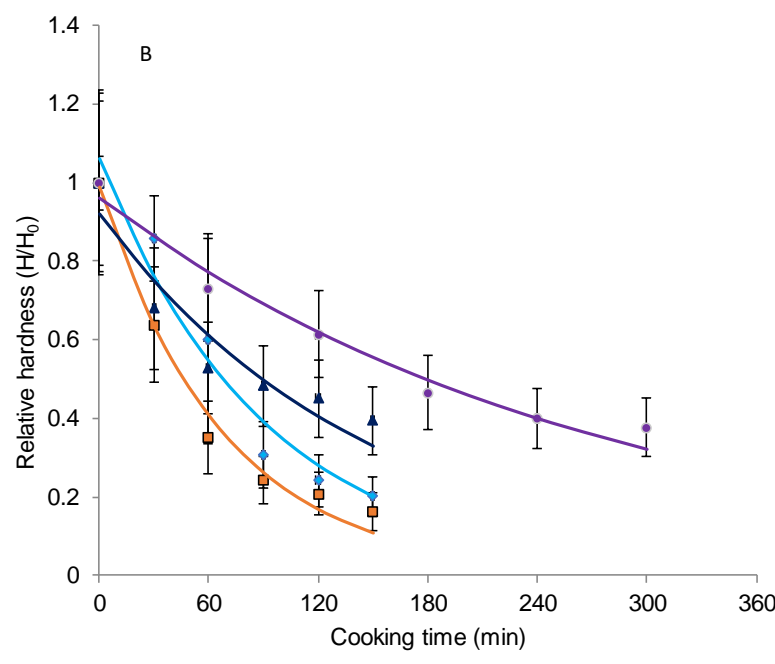

$\square$ Fresh whole beans (control) $\Delta$ whole beans $(11,7 \%$ M.C)
- whole beans (8,1\% M.C) - whole beans (14,5\% M.C)

540 Supplementary Fig. 1. Cooking behavior of cotyledons (A) and whole beans (B) conditioned to 541 different moisture contents through storage above different saturated salt solutions at $35^{\circ} \mathrm{C}$ for 5423 months. Data was modeled following first order kinetics. M.C. is moisture content. 